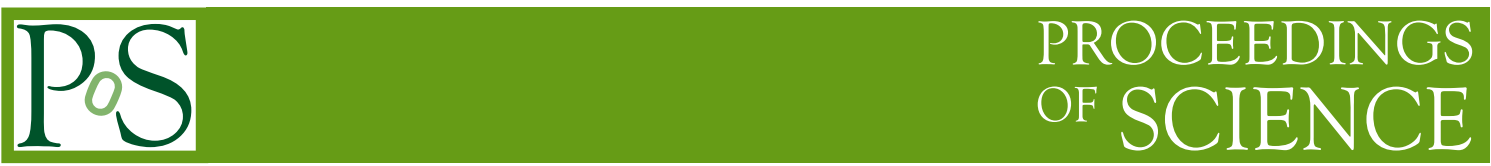

\title{
Semi-annihilating scalar dark matter
}

\author{
Geneviève BELANGÉR, Kristjan KANNIKE*, Alexander PUKHOV and Martti RAIDAL \\ Scuola Normale Superiore di Pisa \\ E-mail: belangerelapth.cnrs.fr, \\ kristjan.kannike@cern.ch, \\ pukhovelapth.cnrs.fr, \\ martti.raidalecern.ch
}

\begin{abstract}
Scalar dark matter models invariant under a discrete $\mathbb{Z}_{3}$ symmetry are studied. Unlike in the usual $\mathbb{Z}_{2}$ case, their phenomenology can contain semi-annihilations - processes in which two dark sector particles scatter into a dark sector and a SM particle. The simplest such model has complex scalar singlet DM stabilised by $\mathbb{Z}_{3}$. Compared to the well-known $\mathbb{Z}_{2}$ case, the new processes can significantly change relic abundance and prospects for direct detection. The requirement that $\mathbb{Z}_{3}$ be not broken spontaneously, however, places a lower bound on the direct detection cross section and will allow the whole parameter space to be tested by XENON1T. Addition of new scalars can stabilise the Higgs potential up to the GUT scale.
\end{abstract}

The European Physical Society Conference on High Energy Physics

18-24 July, 2013

Stockholm, Sweden

${ }^{*}$ Speaker. 


\section{Introduction}

One of the most appealing candidates of dark matter (DM) is a weakly interacting massive particle or WIMP. In this case, DM is a thermal relic whose cosmic density is determined by freeze-out. At high temperature, DM is at thermal equilibrium with the rest of the universe but as the universe expands and cools it begins to annihilate away, but freezes out with further dilution.

What keeps a massive DM particle from decaying into Standard Model (SM) particles? The simplest possible symmetry that prevents DM decay is of course a $\mathbb{Z}_{2}$ parity. However, there is a priori no reason to believe that the symmetry must be $\mathbb{Z}_{2}$. It can very well be $\mathbb{Z}_{3}, \mathbb{Z}_{4}$, or something more complicated. As we shall see, higher $\mathbb{Z}_{N}$ symmetries imply important changes to the phenomenology.

With $\mathbb{Z}_{N}$, where $N$ is larger than two, semi-annihilation processes $x_{i} x_{j} \rightarrow x_{k} X$, where $x_{i}$ is a dark sector particle and $X$ is a SM particle, become possible [1,2,3]. With $\mathbb{Z}_{4}$ and higher, one can have multi-component DM and the so-called DM conversion $x_{i} x_{i} \leftrightarrow x_{j} x_{j}$ becomes possible $[4,5,6]$. This is annihilation, but from one DM component to another.

If a field $\varphi$ has discrete charge $X_{\varphi}$, it transforms under $\mathbb{Z}_{N}$ as

$$
\varphi \rightarrow e^{i \frac{X \varphi}{N} 2 \pi} \varphi
$$

Therefore, addition of charges is modulo $N$ and without loss of generality one can consider charges from 0 to $N-1$.

The $\mathbb{Z}_{N}$ symmetry can come from breaking of a $U(1)$ gauge group that may be embedded in $S O(10)$, for example: $S O(10) \supset U(1)_{X} \rightarrow \mathbb{Z}_{N}$ by a GUT Higgs with $X=N$. However, from the phenomenological point of view, different assignments of discrete charges can give the same low energy potential.

For given field content, there is a limited number of possible terms in the scalar potential due to renormalisability. For higher $N$, the $\mathbb{Z}_{N}$ symmetry approximates the original $U(1)$. In the scalar potential, there is always a $U(1)$ symmetric part and then a few extra terms.

In this talk we look at scalar DM that is made stable by a $\mathbb{Z}_{N}$ symmetry. Scalars are perhaps simplest DM, and they could be seen via their couplings to the Higgs boson. Also, the stability of the Standard Model vacuum is a borderline case [7]. The quartic self-coupling may run to negative values around $10^{10} \mathrm{GeV}$ - this is below the unification scale. It could well be metastable. Adding new scalars to the model can improve the vacuum stability of the scalar potential.

From the purely phenomenological point of view, the simplest model of DM the scalar singlet stabilised by a $\mathbb{Z}_{2}$ symmetry. This model has been studied very thoroughly (see [8] and refs. therein). The model is very constrained because the same coupling that determines the relic density - the quartic coupling of the Higgs and DM - gives the direct detection cross section, Fig.1a,c, proportional to $\lambda_{S H}$.

If the scalar singlet is instead stabilised by $\mathbb{Z}_{3}$, however $[9,10]$, there is a new, cubic term in the scalar potential:

$$
V_{\mathbb{Z}_{3}}=\mu_{H}^{2}|H|^{2}+\lambda_{H}|H|^{4}+\mu_{S}^{2}|S|^{2}+\lambda_{S}|S|^{4}+\lambda_{S H}|S|^{2}|H|^{2}+\frac{\mu_{3}}{2}\left(S^{3}+S^{\dagger 3}\right),
$$

which will induce the semiannihilation reaction given by the Feynman diagram in Fig.1b, proportional to $\lambda_{S H} \mu_{3}$. 


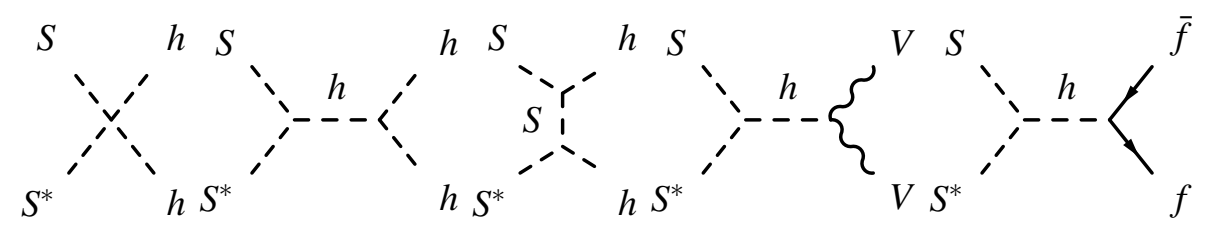

(a)

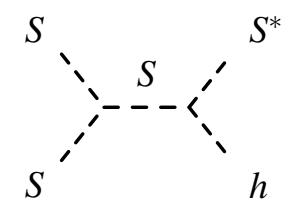

(b)

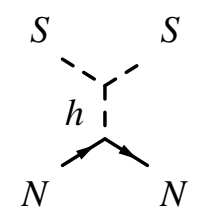

(c)

Figure 1: Feynman diagrams contributing to (a) annihilation and (b) semi-annihilation of DM; and (c) DM cross section with nucleons.

There is a bound on the cubic coupling:

$$
\max \mu_{3} \approx 2 \sqrt{2} \sqrt{\frac{\lambda_{S}}{\delta}} M_{S}
$$

where $0 \leqslant \delta \leqslant 2$ (absolute stability is given by $\delta=2$, while $\delta<2$ gives metastability). Namely, if $\mu_{3}$ is too large, the minimum of the potential where $\mathbb{Z}_{3}$ is conserved is not global. ${ }^{1}$

To compute the relic density we solve the Boltzmann equations with the micrOMEGAs package $[11,12,13,14]$. The equations for the number density, $n$, have been generalised to include semi-annihilation processes

$$
\frac{d n}{d t}=-v \sigma^{S S^{*} \rightarrow X X}\left(n^{2}-\bar{n}^{2}\right)-\frac{1}{2} v \sigma^{S S \rightarrow S^{*} h}\left(n^{2}-n \bar{n}\right)-3 H n,
$$

where $X$ is any SM particle. The treatment of the semi-annihilation term is described in [15] and the fraction of semi-annihilation is defined as

$$
\alpha=\frac{1}{2} \frac{v \sigma^{S S \rightarrow S^{*} h}}{v \sigma^{S S^{*} \rightarrow X X}+\frac{1}{2} v \sigma^{S S \rightarrow S^{*} h}} .
$$

Note that $S S \rightarrow S^{*} h$ is the only semi-annihilation process in this model.

We vary the physical parameters in the ranges allowed by perturbativity and vacuum stability, select points in the experimentally allowed range for the DM relic density.

In Fig. 2 we show the dependence of $\lambda_{S H}$ and $\mu_{3}$ on DM mass. The colour code shows the fraction of semi-annihilation. The narrow black area with $\mu_{3} \approx 0$ corresponds to $\mathbb{Z}_{2} \mathrm{DM}$.

Naïvely it would seem that we could make semi-annihilation arbitrarily large while making $\lambda_{S H}$ arbitrarily small and get a zero annihilation - and direct detection cross section. But as we saw there is a bound on $\mu_{3}$ that depends on DM mass and also on its self-coupling $\lambda_{S}$, shown here for various values. Note that in order to have large semi-annihilation one has to have a rather large self-coupling $\lambda_{S}$ and will require $\mathrm{TeV}$ scale new physics to deal with loss of perturbativity.

\footnotetext{
${ }^{1}$ We have also checked that if we allow metastability, the results practically do not change.
} 

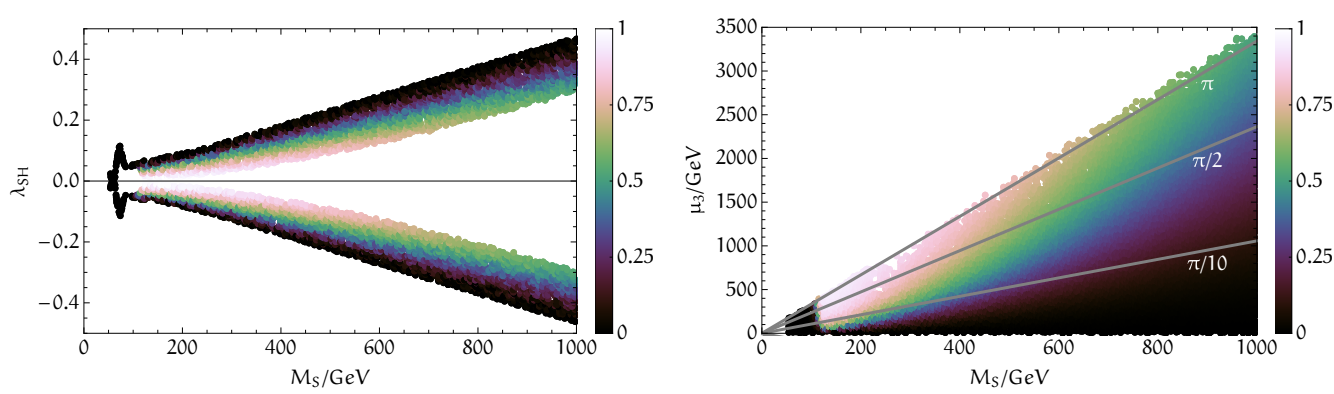

Figure 2: Couplings $\lambda_{S H}$ and $\mu_{3}$ vs. DM mass. Colour code shows fraction of semi-annihilation $\alpha$

In Fig. 3, left, we show the direct detection cross section. In the area at high mass bounded by a white line, the model is valid up to the Grand Unified Theory scale. The larger the semiannihilation, the smaller the cross section, but due to the upper bound (1.3) on $\mu_{3}$, the model can be disproved in XENON1T.
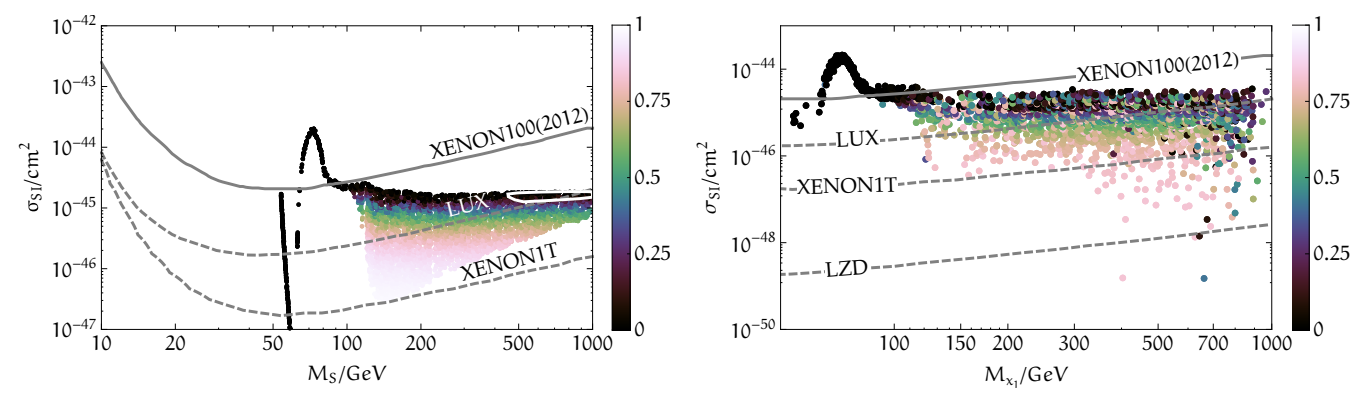

Figure 3: Direct detection in the $\mathbb{Z}_{3}$ models with a singlet (left) and singlet and doublet (right).

What if you want a quartic coupling that gives semi-annihilation? A new, inert doublet has to be added to the model [15]. The scalar potential is

$$
\begin{aligned}
V_{\mathbb{Z}_{3}} & =\mu_{1}^{2}\left|H_{1}\right|^{2}+\lambda_{1}\left|H_{1}\right|^{4}+\mu_{2}^{2}\left|H_{2}\right|^{2}+\lambda_{2}\left|H_{2}\right|^{4}+\mu_{S}^{2}|S|^{2}+\lambda_{S}|S|^{4}+\lambda_{S 1}|S|^{2}\left|H_{1}\right|^{2} \\
& +\lambda_{S 2}|S|^{2}\left|H_{2}\right|^{2}+\lambda_{3}\left|H_{1}\right|^{2}\left|H_{2}\right|^{2}+\lambda_{4}\left(H_{1}^{\dagger} H_{2}\right)\left(H_{2}^{\dagger} H_{1}\right)+\frac{\mu_{S}^{\prime \prime}}{2}\left(S^{3}+S^{\dagger 3}\right) \\
& +\frac{\lambda_{S 12}}{2}\left(S^{2} H_{1}^{\dagger} H_{2}+S^{\dagger 2} H_{2}^{\dagger} H_{1}\right)+\frac{\mu_{S H}}{2}\left(S H_{2}^{\dagger} H_{1}+S^{\dagger} H_{1}^{\dagger} H_{2}\right),
\end{aligned}
$$

The singlet $S$ and the doublet $H_{2}$ mix into $x_{1}$ and $x_{2}$ with a very small mixing angle, to avoid large coupling to the $Z$ boson. $x_{2}$ decays into $x_{1}$ since they have the same discrete charge.

Now, besides the several other new couplings, there is the quartic semi-annihilation coupling $\lambda_{S 12}$, giving rise to a new semi-annihilation process $x_{1} x_{1} \rightarrow x_{2} h$. The Higgs-DM coupling behaves similarly, but now there is semi-annihilation also at large masses $[15,16]$, see Fig. 3, right. The direct detection cross section goes even lower, but again one can expect the scalar couplings to go non-perturbative at a not too high scale in this case.

The $h \rightarrow \gamma \gamma$ rate in this model in Fig. 4 is compatible with the experimental limits [17]. 


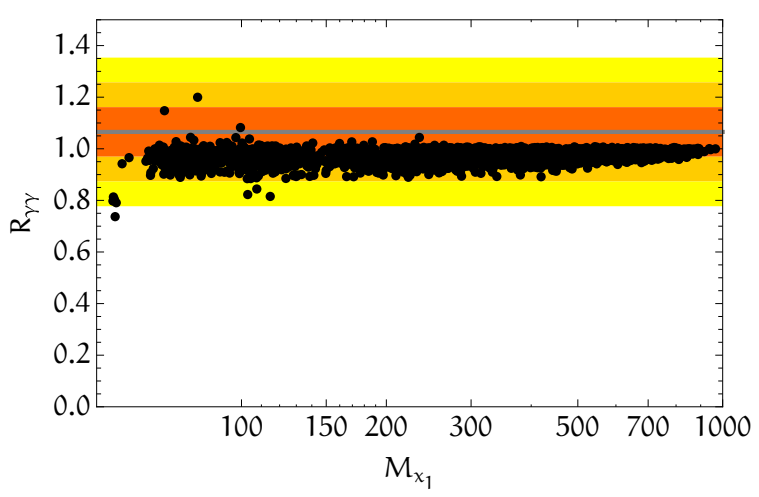

Figure 4: $h \rightarrow \gamma \gamma$ rate.

\section{References}

[1] F. D'Eramo and J. Thaler, JHEP 1006 (2010) 109 [arXiv:1003.5912 [hep-ph]].

[2] T. Hambye, JHEP 0901 (2009) 028 [arXiv:0811.0172 [hep-ph]].

[3] T. Hambye and M. H. G. Tytgat, Phys. Lett. B 683 (2010) 39 [arXiv:0907.1007 [hep-ph]].

[4] Z. -P. Liu, Y. -L. Wu and Y. -F. Zhou, Eur. Phys. J. C 71 (2011) 1749 [arXiv:1101.4148 [hep-ph]].

[5] G. Belanger and J. -C. Park, JCAP 1203 (2012) 038 [arXiv:1112.4491 [hep-ph]].

[6] A. Adulpravitchai, B. Batell and J. Pradler, Phys. Lett. B 700 (2011) 207 [arXiv:1103.3053 [hep-ph]].

[7] D. Buttazzo, G. Degrassi, P. P. Giardino, G. F. Giudice, F. Sala, A. Salvio and A. Strumia, arXiv:1307.3536 [hep-ph].

[8] J. M. Cline, K. Kainulainen, P. Scott and C. Weniger, Phys. Rev. D 88 (2013) 055025 [arXiv:1306.4710 [hep-ph]].

[9] E. Ma, Phys. Lett. B 662 (2008) 49 [arXiv:0708.3371 [hep-ph]].

[10] G. Belanger, K. Kannike, A. Pukhov and M. Raidal, JCAP 1301 (2013) 022 [arXiv:1211.1014 [hep-ph]].

[11] G. Belanger, F. Boudjema, A. Pukhov and A. Semenov, Comput. Phys. Commun. 176 (2007) 367 [hep-ph/0607059].

[12] G. Belanger, F. Boudjema, A. Pukhov and A. Semenov, Comput. Phys. Commun. 180 (2009) 747 [arXiv:0803.2360 [hep-ph]].

[13] G. Belanger, F. Boudjema, P. Brun, A. Pukhov, S. Rosier-Lees, P. Salati and A. Semenov, Comput. Phys. Commun. 182 (2011) 842 [arXiv:1004.1092 [hep-ph]].

[14] G. Belanger, F. Boudjema, A. Pukhov and A. Semenov, arXiv:1305.0237 [hep-ph].

[15] G. Belanger, K. Kannike, A. Pukhov and M. Raidal, JCAP 1204 (2012) 010 [arXiv:1202.2962 [hep-ph]].

[16] G. Belanger, K. Kannike, A. Pukhov and M. Raidal, in progress.

[17] P. P. Giardino, K. Kannike, I. Masina, M. Raidal and A. Strumia, arXiv:1303.3570 [hep-ph]. 